\title{
Video Article \\ Preparation of Hollow Polystyrene Particles and Microcapsules by Radical Polymerization of Janus Droplets Consisting of Hydrocarbon and Fluorocarbon Oils
}

\author{
Yutaka Takahashi ${ }^{1}$, Mari Kano ${ }^{1}$, Natsu Yanagisawa ${ }^{1}$, Yukishige Kondo ${ }^{1}$ \\ ${ }^{1}$ Department of Industrial Chemistry, Faculty of Engineering, Tokyo University of Science \\ Correspondence to: Yukishige Kondo at ykondo@rs.tus.ac.jp
}

URL: https://www.jove.com/video/56922

DOI: doi:10.3791/56922

Keywords: Chemistry, Issue 131, Hollow particles, Microcapsules, Radical polymerization, Janus oil droplets, Fluorocarbon oils, Emulsions

Date Published: 1/25/2018

Citation: Takahashi, Y., Kano, M., Yanagisawa, N., Kondo, Y. Preparation of Hollow Polystyrene Particles and Microcapsules by Radical Polymerization of Janus Droplets Consisting of Hydrocarbon and Fluorocarbon Oils. J. Vis. Exp. (131), e56922, doi:10.3791/56922 (2018).

\section{Abstract}

In this article, we have demonstrated a method for producing hollow particles and microcapsules using oil droplets consisting of hydrocarbon oil (styrene) and fluorocarbon oil (perfluoro- $n$-octane, PFO) in aqueous surfactant (sodium dodecylsulfate, SDS) solutions. Since fluorocarbon oils are immiscible with hydrocarbon oils, the two oils are separated. Emulsions are prepared by stirring styrene/PFO/aqueous SDS solution mixtures at $80^{\circ} \mathrm{C}$. The type of emulsions and the morphology of droplets in the emulsions are observed by light microscope and scanning confocal fluorescence microscope. It is found that oil droplets with Janus-type morphologies consisting of mutually immiscible styrene and PFO are formed in aqueous SDS solutions. Polystyrene particles are fabricated by radical polymerization of the ternary mixtures of styrene/PFO/ aqueous SDS solution at $80^{\circ} \mathrm{C}$. The morphologies of the polystyrene are confirmed by scanning electron microscopy and scanning transmission electron microscopy observations. These observations show the preparation of hollow polystyrene particles with a single hole on the surface. To our knowledge, this method is a novel strategy using the immiscibility of hydrocarbon and fluorocarbon oils. The hollow particles can also be applied to the preparation of microcapsules.

\section{Video Link}

The video component of this article can be found at https://www.jove.com/video/56922/

\section{Introduction}

While spherical polymer particles have been widely used in various industrial applications, it is well-known that dimple particles, hemispheres, disks, and ellipsoids, have been prepared by seeded polymerizations $s^{1,2,3}$, photo-polymerization of non-spherical monomer droplets using microreactor with specific geometries ${ }^{4,5}$, self-organization using reprecipitation of polymers ${ }^{6}$, and deformation of spherical polymer particles by mechanical external forces ${ }^{7,8}$. In particular, hollow polymer particles with micrometer-size have been fabricated by evaporation of a good solvent from spherical polymer particles swollen by the solvent ${ }^{9,10}$ and polymerization using multiple emulsions ${ }^{11,12}$.

In this work, we focus on the usage of mutual immiscibility of hydrocarbon and fluorocarbon oils in the fabrication of polymer particles. Hybrid surfactants have a hydrocarbon chain and a fluorocarbon chain in the molecule. Previously, we have reported the unique properties of hybrid surfactants that are not observed in conventional surfactants ${ }^{13,14,15}$. We have also studied emulsions using hydrocarbon oil, fluorocarbon oil, and aqueous surfactant solution, which are mutually immiscible ${ }^{16}$. However, there are very few studies of the emulsions ${ }^{17}$. These studies have described the morphology of oil droplets consisting of hydrocarbon oil and fluorocarbon oil in aqueous surfactant solutions.

Here, we show a detailed protocol of the fabrication of hollow polymer particles by radical polymerization using oil droplets in emulsions consisting of hydrocarbon oil, fluorocarbon oil, and aqueous sodium dodecylsulfate (SDS) solutions. We suggest a new strategy, which is different from conventional methods, for the preparation of non-spherical polymer particles. This method can simply fabricate the hollow polymer particles in a short time. In addition, the protocol of the preparation of microcapsules through the hollow polymer particles is shown.

\section{Protocol}

Caution: Please wear a lab-coat, gloves, and safety glasses and read material safety data sheets (MSDS) before use. All purchased materials have been used without further purification.

\section{Preparation of Emulsions Consisting of Styrene/PFO/Aqueous SDS Solution Mixtures}

1. To prepare $5 \mathrm{mM}$ of aqueous SDS solution, dissolve $14.5 \mathrm{mg}$ of SDS in $10 \mathrm{~mL}$ of high-purity $\mathrm{H}_{2} \mathrm{O}$. NOTE: High-purity $\mathrm{H}_{2} \mathrm{O}$ has been used (resistivity $(R)=18 \mathrm{M} \Omega \mathrm{cm}$, surface tension $(\gamma)=72.0 \mathrm{mN} \mathrm{m}^{-1}$ at $25^{\circ} \mathrm{C}$ ). 
2. Add $1.5 \mathrm{~g}$ of styrene, $0.6 \mathrm{~g}$ of PFO, and $0.9 \mathrm{~g}$ of $5 \mathrm{mM}$ of aqueous SDS solution to a $10 \mathrm{~mL}$ glass vial with a stir bar. NOTE: In scanning confocal fluorescence microscopy observations, add $2.6 \mathrm{mg}$ of coumarin 102 and $0.062 \mathrm{mg}$ of calcein to the mixture.

3. Stir the mixture for $60 \mathrm{~min}$ at $1,150 \mathrm{rpm}$ under room temperature and then raise the temperature to $80^{\circ} \mathrm{C}$.

4. Stir the mixture for $60 \mathrm{~min}$ at $1,150 \mathrm{rpm}$ and $80^{\circ} \mathrm{C}$

\section{Fabrication of polymer particles using Emulsions Consisting of Styrene/PFO/Aqueous SDS Solution Mixtures}

1. Add $3.9 \mathrm{mg}$ of potassium peroxodisulfate, $2 \mathrm{mg}$ of pyrene, $1.5 \mathrm{~g}$ of styrene, $0.6 \mathrm{~g}$ of PFO, and $0.9 \mathrm{~g}$ of $5 \mathrm{mM}$ of aqueous SDS solution to a 10 $\mathrm{mL}$ glass vial with a stir bar and seal it with a rubber septum.

2. Deoxygenate the mixture by bubbling nitrogen gas through a syringe needle for $30 \mathrm{~min}$. Caution: Slowly flow the nitrogen gas to not generate a large amount of foam.

3. To prepare the emulsion, stir the mixture for $60 \mathrm{~min}$ at $1,150 \mathrm{rpm}$ and room temperature and then raise the temperature to $80{ }^{\circ} \mathrm{C}$.

4. Stir the mixture for $30 \mathrm{~min}$ at $1,150 \mathrm{rpm}$ and $80^{\circ} \mathrm{C}$.

5. Transfer the cloudy part $(1.8 \mathrm{~mL})$ in the resultant solution $(3 \mathrm{~mL})$ to the test tube and then add $30 \%$ aqueous ethanol solution to completely terminate the polymerization reaction.

6. Sonicate (Power: $130 \mathrm{~W}$, Frequency: $4.2 \mathrm{kHz}$ ) for $10 \mathrm{~min}$ to wash resultant polymer particles and then centrifuge for $10 \mathrm{~min}$ at $2300 \mathrm{x} \mathrm{g}$.

7. To obtain the polymer particles, remove the supernatant solution from the test tube.

8. Add $3 \mathrm{~mL}$ of water to the resultant solids in the test tube. Sonicate (Power: $130 \mathrm{~W}$, Frequency: $4.2 \mathrm{kHz}$ ) for 10 min and centrifuge for $10 \mathrm{~min}$ at $2300 \mathrm{x}$ g. Remove the supernatant solution from the test tube.

9. Repeat the washing procedure (Step 2.8) until foam is not generated from the supernatant solution. Evaporate water to obtain the solid of hollow polymer particles.

\section{Preparation of microcapsules using the hollow polymer particles}

1. Add $1 \mathrm{mg}$ of hollow polystyrene particles (step 2.9) and $4 \mathrm{~mL}$ of water to a $10 \mathrm{~mL}$ glass vial with a stir bar.

2. Sonicate (Power: $130 \mathrm{~W}$, Frequency: $4.2 \mathrm{kHz}$ ) for $10 \mathrm{~min}$ to disperse the hollow particles in water.

3. Add $0.1 \mathrm{~mL}$ of toluene to the water dispersed the particles and then stir it for $1 \mathrm{~h}$ at $100 \mathrm{rpm}$ under room temperature. Transfer the liquid to a test tube.

Caution: If the rotation per minute increases, the morphology of particles will deform.

4. Centrifuge the liquid for $10 \mathrm{~min}$ at $2300 \mathrm{xg}$ to isolate the microcapsules form it. Remove the supernatant solution from the test tube.

\section{Representative Results}

Light and scanning confocal fluorescence microscopy observations were performed to determine the morphology and composition of droplets in the emulsions consisting of styrene, PFO, and $5 \mathrm{mM}$ aqueous SDS solution (Figure 1). Scanning electron microscopy (SEM) and scanning transmission electron microscopy (STEM) observations showed the formation of hollow particles and microcapsules (Figure 2)

The DIC and confocal fluorescence microscopy images of emulsions consisting of ternary mixtures of styrene/PFO/aqueous SDS solution indicate that the continuous phase is aqueous SDS solution, because green fluorescence corresponding to calcein was observed in the continuous phase, and therefore, oil-in-water type emulsions are formed (Figure 1a and 1b). These images also show that the oil droplets consist of styrene and PFO, which are mutually immiscible. The DIC and confocal fluorescence microscopy images of emulsions containing coumarin 102 reveal that a PFO droplet is located at the interface between water and a styrene droplet. The ternary mixtures of styrene, PFO, and aqueous SDS solution at $5 \mathrm{mM}$ form emulsions containing Janus oil droplets, which consist of styrene and PFO.

The SEM and STEM images of polymer particles fabricated by the radical polymerization of the emulsions of styrene/PFO/5 mM aqueous SDS solution show the preparation of hollow non-spherical polystyrene particles with a hole on the surface (Figure $\mathbf{2 a}$ and $\mathbf{2 b}$ ). The formation rate, diameter, and hole size of the hollow polystyrene particles are evaluated by observing 200 particles through SEM and STEM. The averaged diameter estimated from SEM observations is $1.3 \mu \mathrm{m}$. The size is consistent with the diameter of the polymer particles estimated from dynamic light scattering measurements. The formation rate is approximately $100 \%$. The averaged hole-size and volume of the hole in the hollow particles are $0.8 \pm 0.4 \mu \mathrm{m}$ and $0.9 \pm 0.4 \mu \mathrm{m}^{3}$, respectively. Therefore, the hollow polystyrene particles having a $0.8 \mu \mathrm{m}$ hole were fabricated by radical polymerization of Janus droplets containing styrene in the emulsions. 


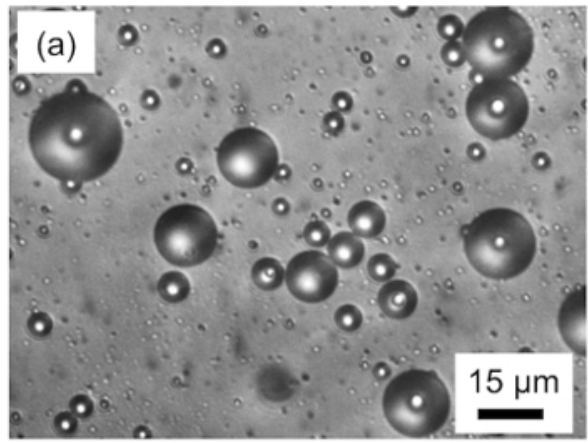

\section{(b)}
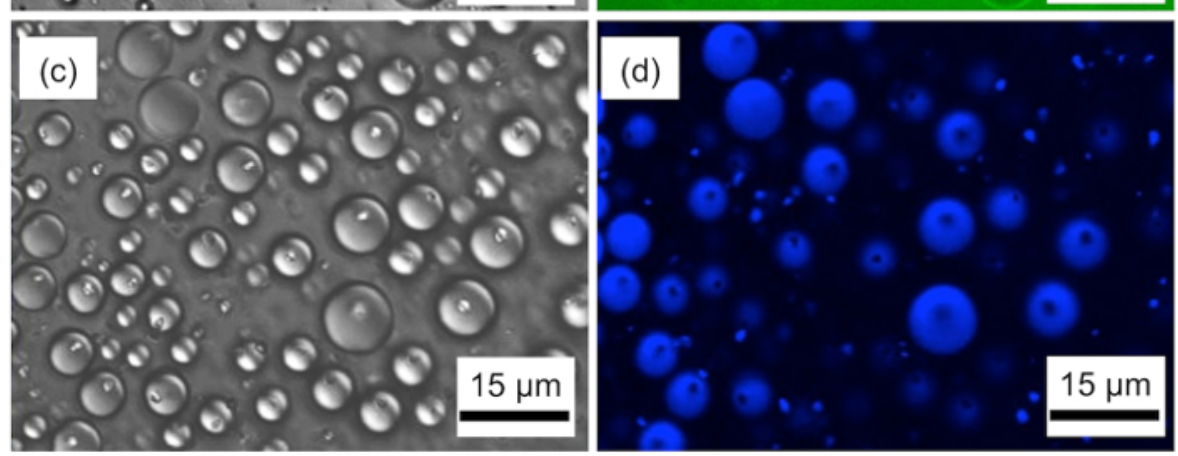

Figure 1. Morphologies of droplets in emulsions consisting of styrene, PFO, and $5 \mathrm{mM}$ aqueous SDS solution. (a) Differential interference contrast (DIC) and (b) confocal fluorescence microscopy images of the emulsions containing aqueous fluorescent calcein. (c) DIC and (d) confocal fluorescence microscopy images of the emulsions containing oil-soluble fluorescent coumarin 102. Please click here to view a larger version of this figure.
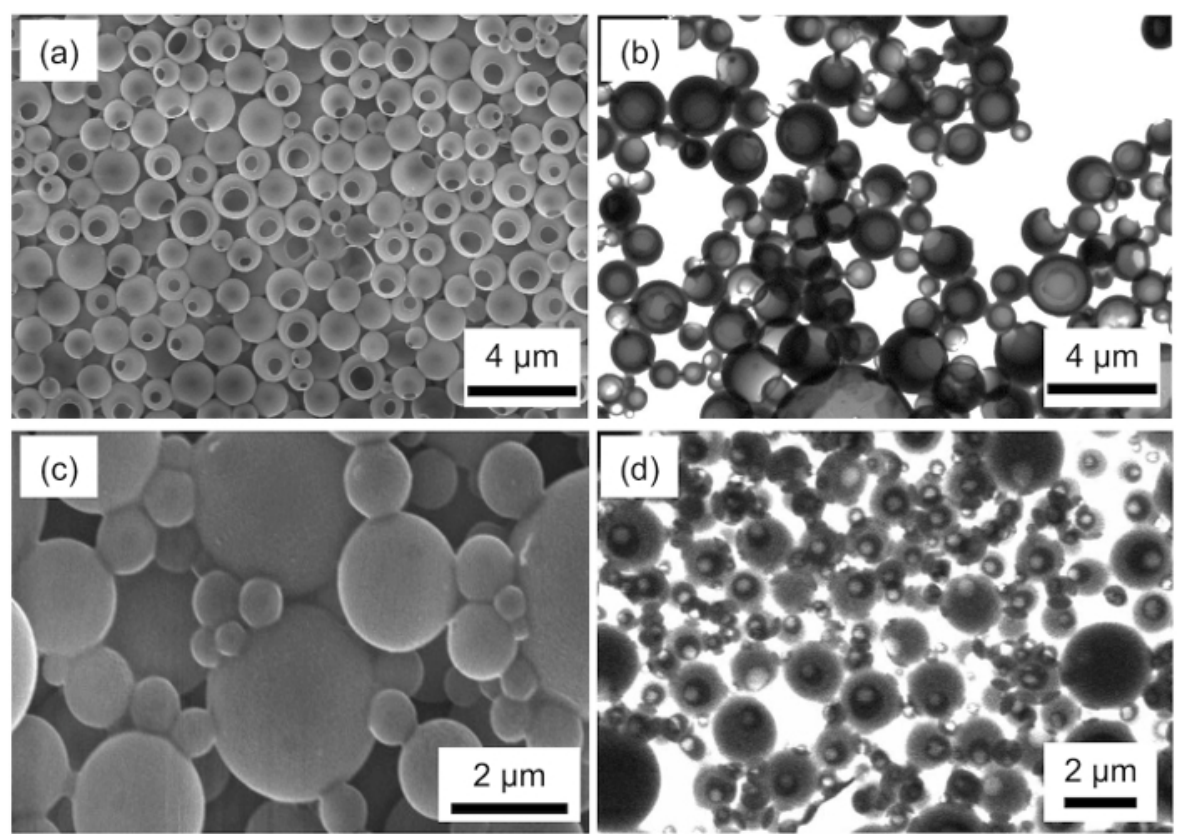

Figure 2. Morphologies of hollow polystyrene particles and microcapsules fabricated using emulsions consisting of styrene, PFO, and $5 \mathrm{mM}$ aqueous SDS solution. (a) SEM and (b) STEM images of the hollow polystyrene particles. (c) SEM and (d) STEM images of the microcapsules. Please click here to view a larger version of this figure.

\section{Discussion}

The emulsions containing Janus droplets of styrene and PFO were obtained above $10 \mathrm{wt} \%$ of the weight fraction of aqueous SDS concentration and any weight fraction of styrene and PFO. When the various weight-fractions of the emulsions containing the Janus droplets have been polymerized for $30 \mathrm{~min}$, the hollow polystyrene particles can be obtained for all composition. These results indicate that the protocol demonstrated here is simple. In addition, the diameter, hole size, and hole volume of the hollow polystyrene particles can be controlled by the polymerization time in the method described here. 
We suggest the following mechanism for the fabrication of the hollow polystyrene particles. Janus oil droplets consisting of styrene and PFO formed in the O/W emulsions containing mixtures of aqueous SDS solution, styrene, and PFO at $80^{\circ} \mathrm{C}$. PFO droplets are located on the surface of styrene droplets. Since KPS, an initiator of the polymerization, is soluble in the water phase, the polymerization will progress at the interface of the styrene/aqueous SDS solution. Styrene in the ternary emulsions was polymerized to polystyrene at $80^{\circ} \mathrm{C}$, whereas PFO, having no polymerizable groups, remained in the emulsions at that temperature. After the polymerization was terminated, a hole on the polystyrene particle was formed by removal of PFO. Therefore, the hollow cup-type polystyrene particles having a hole on the surface were fabricated atany weight fraction of styrene and PFO and all polymerization times.

Adding a small amount of toluene to the hollow polystyrene particles fabricated by radical polymerization for 90 min brings about sealing the holes on the hollow polystyrene particles. This phenomena is consistent with that reported by Hyuk et al. ${ }^{9}$. The capsulation method can contain an aqueous material in the particles.

In this article, we have demonstrated a fabrication method of hollow polymer particles using Janus oil droplets consisting of styrene and PFO, which are mutually immiscible. The preparation method of non-spherical polymer particles has already been studied because of their potential use in various applications. This strategy using Janus oil droplets of hydrocarbon oil and fluorocarbon oil will apply to the fabrication of nonspherical polymer particles from various types of monomer and drug delivery system.

\section{Disclosures}

We have nothing to disclose.

\section{Acknowledgements}

We have no acknowledgements.

\section{References}

1. Okubo, M., Fujibayashi, T., \& Terada, A. Synthesis of micron-sized, monodisperse polymer particles of disc-like and polyhedral shapes by seeded dispersion polymerization. Colloid Polym Sci. 283 (7), 793-798 (2005).

2. Fujibayashi, T., \& Okubo, M. Preparation and Thermodynamic Stability of Micron-Sized, Monodisperse Composite Polymer Particles of Disclike Shapes by Seeded Dispersion Polymerization. Langmuir. 23 (15), 7958-7962 (2007).

3. Okubo, M., Fujibayashi, T., Yamada, M., \& Minami, H. Micron-sized, monodisperse, snowman/confetti-shaped polymer particles by seeded dispersion polymerization. Colloid Polym Sci. 283 (9), 1041-1045 (2005).

4. Xu, S. et al. Generation of Monodisperse Particles by Using Microfluidics: Control over Size, Shape, and Composition. Angew Chem Int Ed. 44 (5), 724-728 (2005).

5. Serra, C. A., \& Chang, Z. Microfluidic-Assisted Synthesis of Polymer Particles. Chem Eng Tech. 31 (8), $1099-1115$ (2008).

6. Higuchi, T., Yabu, H., \& Shimomura, M. Simple preparation of hemispherical polystyrene particles. Colloids Surf A. 284 250-253 (2006).

7. Sun, Z. Q. et al. Nonspherical Colloidal Crystals Fabricated by the Thermal Pressing of Colloidal Crystal Chips. Langmuir. 21 (20), $8987-8991$ (2005).

8. Deng, Y. et al. A novel approach to the construction of 3-D ordered macrostructures with polyhedral particles. J Mater Chem. 18 (4), $408-415$ (2008).

9. Hyuk Im, S., Jeong, U., \& Xia, Y. Polymer hollow particles with controllable holes in their surfaces. Nat Mater. 4 (9), $671-675$ (2005)

10. Saito, N., Kagari, Y., \& Okubo, M. Effect of Colloidal Stabilizer on the Shape of Polystyrene/Poly(methyl methacrylate) Composite Particles Prepared in Aqueous Medium by the Solvent Evaporation Method. Langmuir. 22 (22), 9397-9402 (2006).

11. Cai, P.-j., Tang, Y.-j., Wang, Y.-t., \& Cao, Y.-j. Fabrication of polystyrene hollow spheres in W/O/W multiple emulsions. Mater Chem Phys. 124 (1), 10-12 (2010).

12. Liang, S.-S., Chen, S.-L., \& Chen, S.-H. Diverse macroporous spheres synthesized by multiple emulsion polymerization for protein analyses. Chem Commun. 47 (29), 8385-8387 (2011).

13. Kondo, Y., \& Yoshino, N. Hybrid fluorocarbon/hydrocarbon surfactants. Curr Opin Colloid Interface Sci. 10 (3-4), 88-93 (2005).

14. Takahashi, Y., Kondo, Y., Schmidt, J., \& Talmon, Y. Self-Assembly of a Fluorocarbon-Hydrocarbon Hybrid Surfactant: Dependence of Morphology on Surfactant Concentration and Time. J Phys Chem B. 114 (42), 13319-13325 (2010).

15. Takahashi, Y., Nasu, Y., Aramaki, K., \& Kondo, Y. Unusual viscoelastic behavior of aqueous solutions of fluorocarbon-hydrocarbon hybrid surfactant and its morphological transformations. J Fluor Chem. 145 (0), 141-147 (2013).

16. Yoshino, N. et al. Syntheses of Hybrid Anionic Surfactants Containing Fluorocarbon and Hydrocarbon Chains. Langmuir. 11 (2), $466-469$ (1995).

17. Zarzar, L. D. et al. Dynamically reconfigurable complex emulsions via tunable interfacial tensions. Nature. 518 (7540), $520-524$ (2015). 